\title{
THE INCIDENCE OF RISK UNDER CREDIT INSURANCE
}

\author{
BY GREG TAYLOR \\ December 1991 \\ Revised May 1993 \\ A Coopers \& Lybrand research report
}

\begin{abstract}
The incidence of risk under a credit insurance policy depends on the original term of the policy and the policy duration at which the incidence of risk is considered. Section 3 of the paper describes the procedure used to fit a bivariate function to this incidence. Section 4 gives the numerical detail of this model. Section 5 makes a comparison of the model with the data from which it was developed. Section 6 adds some general comments.
\end{abstract}

KEYWORDS

Credit insurance; incidence of risk; claim frequency.

\section{INTRODUCTION}

Credit insurance provides coverage of insureds who are subject to obligations to repay credit advances by means of periodic instalments. Coverage may be provided against the events of sickness/accident and/or unemployment.

Often, the credit to be insured will take the form of a fixed term loan, such as under a hire purchase contract. In this event the credit insurance policy would usually have a fixed term matching that of the credit contract. Typically, the fixed terms vary between 6 and 84 months, most commonly 24 or 36 months. Terms outside the range 6 to 84 months are occasionally encountered.

Typically, a single premium is paid at inception of the policy. It is then necessary for the insurer to spread this premium over the term of the policy for premium earning purposes. As the policies are of rather longer term than the conventional one-year coverages provided in the non-life market, it is by no means evident that premiums should be earned at a uniform rate over the whole term of a policy. Indeed, experience with this type of insurance invariably indicates a concentration of the risk of claim under the policy in the early part of the term.

The construction of a premium earning formula therefore involves the modelling of the incidence of risk over the term of such a policy. Although this is not particularly difficult in relation to any particular policy term, each separate policy term requires its own model, as will be seen in Section 4. There 
is, therefore, the potential for one to finish with an inelegant plethora of premium earning models.

Section 4 illustrates how this situation may be averted. The final model there contains only a handful of parameters and deals with policies of any term.

\section{DATA AND NOTATION}

Data were provided in respect of the fixed term policies of an Australian credit insurer.

The insurer provided an analysis of its data base giving, separately for each policy term, a tabulation of:

(a) number of claims, subdivided simultaneously according to:

(i) month of attachment of the policy generating the claim;

(ii) the development month (i.e. the number of months after month of attachment) of occurrence of the claim;

and

(b) number of premium transactions, subdivided simultaneously according to:

(i) month of attachment of the policy generating the premium transaction;

(ii) the development month of the premium transaction.

A "premium transaction" is any payment of premium by the insured, refund of premium to the insured, or error correcting journal entry by the insurer. A dissection of recorded transactions according to these categories was not available. For the purpose of the present study, and subject to the qualifications below, the transactions of development month 0 (i.e. the month of attachment) were taken to count 1 for each new premium, whereas those of subsequent development months to count 1 for each premium refund in respect of policy cancellation. The implications of this interpretation for the analysis are pointed out in Section 3.1.

As a consequence of a transfer of the portfolio from one insurer to another, the data base contained:

(a) no information in respect of claims which occurred more than two years prior to the transfer;

(b) incomplete information in respect of policies effected more than two years prior to the transfer.

As a result, there was incomplete experience at policy durations in excess of 39 months. As will be seen in the graphs of Section 5, however, this proved to be a relatively minor shortcoming of the data, since few claims occur at such high durations.

Let

$p_{i j}^{t}=$ number of premium transactions in development month $j$ of policies with month of attachment $i$ and policy term $t$ months; 
$N_{i j}^{t}=$ number of claims notified to date in respect of policies with month of attachment $i$ and policy term $t$ months, and with date of occurrence within development month $j$.

Note that $N_{i j}^{t}$ includes only claims notified to date. It follows that more recent development months will involve a systematic under-statement of the number of claims incurred in those months. Collateral data indicate that the great majority of claims within this line of business are reported within a few months. Thus, provided that the most recent few months of experience are excluded from consideration, any under-estimation of claim frequencies should be relatively small. Detail of the manner in which this question was treated is given in Section 3.1.

\section{ANALYSIS}

\subsection{Observed incidence of risk}

The term incidence of risk has been used to this point without strict definition. It is now defined.

Let

$$
e_{i j}^{t}=p_{i 0}^{t}-\sum_{k=1}^{j-1} p_{i k}^{t}-1 / 2 p_{i j}^{t}
$$

According to the interpretation of the recorded premium transactions $p_{i j}^{t}$ set out in Section 2, $e_{i j}^{t}$ gives the average number of policies, with month of attachment $i$ and policy term $t$, which remain in force during development month $j$. Equation (3.1) involves an implicit assumption that cancellations occur uniformly over development months.

Since the interpretation of the $p_{i 0}^{t}$ effectively ignores cancellations in the month of attachment, any such cancellations will lead to overstatement of $e_{i j}^{i}$ for all $j$. This effect was thought to be small.

Correction journals in month of attachment would tend to overstate $e_{i j}^{i}$ for all $j$, while those in development month $k(>0)$ would tend to understate $e_{i j}^{t}$ for all $j \geq k$. Such corrections are generally concentrated in the early development months. To the extent that over- and under-statements fail to balance, there will be a systematic over- or under-statement of $e_{i j}^{t}$ for the higher values of $j$ (for given $i$ and $t$ ).

To the extent that policy cancellations are also biased towards the front end of policy term, implying approximately constant exposure at the higher development months, the net result of the errors in computation of policies in force will be a proportionately constant over- or under-statement of claim frequency $\lambda_{j}^{t}$, defined below, at the higher values of $j$. This has little effect on the incidence of risk, as defined below.

To the extent that policy cancellations are more dispersed over development months than are correction journals, the approximately constant error arising from the latter at higher $j$ will relate to an exposure which reduces with 
increasing $j$. The under- or over-statement in exposure will therefore grow if relative terms as $j$ increases. Correspondingly, the error in incidence of risk, as defined below, will also grow in relative terms.

It was not possible to correct the data for such effects, but they were thought to be relatively small.

Let

$I_{i j}^{t}=$ number of claims incurred (i.e. notified to date $\left(N_{i j}^{t}\right)+$ IBNR) in respect of policies with month of attachment $i$ and policy term $t$ months, and with date of occurrence within development month $j$;

and suppose that

$$
E\left[I_{i j}^{t}\right]=e_{i j}^{i} \lambda_{j}^{t},
$$

where $\lambda_{j}^{t}$ is evidently a claim frequency parameter applying to development month $j$ within policy term $t$. Thus claims will be expected to occur (by number) over the term of the policy in proportion with $\lambda_{j}^{t}$.

In the particular portfolio considered here, there was little variation in claim size as $j$ varied. The majority of claims involved sickness, injury or unemployment of only a few months. Unemployment coverage was so limited by terms of the insurance. As a result, tabulations of average claim sizes by $j$ (for specific $i$ and $t$ ) yielded no discernible trends until $j$ reached values close to $t$ (about $t-1$ or $t-2$ ), when average claim sizes were reduced. Claim frequencies are generally small at these values of $j$, and so this variation of claim size has been ignored.

With no trend in claim size as $j$ varies, amounts of claims will be incurred in proportion with $\lambda_{j}^{t}$. The distribution

$$
\lambda_{j}^{t} \mid \sum_{k=0}^{t} \lambda_{k}^{t}
$$

will be referred to as the incidence of risk over policy term $t$. Premium should be spread over the term of the policy in these proportions for earning purposes.

Let $\lambda_{j}^{t}$ be estimated by:

$$
\hat{\lambda}_{i j}^{t}=N_{i j}^{t} / e_{i j}^{t},
$$

which provides a separate estimator of $\lambda_{j}^{t}$ for each month of attachment $i$. It may be calculated that:

$$
E\left[\hat{\lambda}_{i j}^{t}\right]=\lambda_{j}^{t}\left(N_{i j}^{t} / I_{i j}^{t}\right)
$$

the bracketed term on the right indicating the factor by which the estimator (3.3) is biased. As noted above, the bias tends to be small:

$$
N_{i j}^{t} / I_{i j}^{t} \sim 1,
$$

provided that the development month $j$ is not too close to the end of the period of investigation. 
Accordingly, the estimator (3.3) should either be used only for those values of $j$ sufficiently separated from the end of the investigation, or should be replaced by:

$$
\hat{\hat{\lambda}}_{i j}^{t}=\hat{\lambda}_{i j}^{t} \hat{\theta}_{i j} \text {, }
$$

where $\hat{\theta}_{i j}$ is an unbiased estimator of $E\left[I_{i j}^{t} / N_{i j}^{t}\right]$. Combination of (3.4) and (3.6) then yields that

$$
\hat{\hat{\lambda}}_{i j}^{t}
$$

is an unbiased estimator of $\lambda_{j}^{t}$ provided that the random variables $N_{i j}^{t}$ and $I_{i j}^{t} / N_{i j}^{t}$ can be assumed stochastically independent.

In order to avoid notational inconvenience, the following Sections will use $\hat{\lambda}_{i j}^{t}$ to denote either of the estimators of $\lambda_{i j}^{t}$ defined in (3.3) and (3.6).

Examination of values of $\hat{\lambda}_{i j}^{t}$ for $j$ and $t$ fixed but $i$ varying revealed no trend in claim frequency with month of occurrence (actually quarter of occurrence was used in the investigation described here). Hence, these estimators for different values of $i$ were collapsed into a single estimator independent of $i$ :

$$
\hat{\lambda}_{j}^{t}=\sum_{i} \hat{\lambda}_{i j}^{t} / m_{j}^{t},
$$

where $m_{j}^{t}$ is the number of periods of occurrence included in the averaging. It would be possible to replace (3.7) by some form of weighted average if this were seen as desirable.

\subsection{Model of incidence of risk}

The estimators (3.7) depend on both policy term $t$ and development month $j$. As the graphs of Section 5 demonstrate, the parameter $\lambda_{j}^{t}$ is genuinely bivariate. That is, its shape as a function of $j$ changes with $t$; similarly its shape as a function of $t$ changes with $j$.

In order to avoid an over-abundance of parameter estimates, it is necessary to estimate $\lambda_{j}^{t}$ simultaneously as a function of $t$ and $j$. Preliminary plots of $\hat{\lambda}_{j}^{t}$ against $j$ for various fixed values of $t$ indicated that $\lambda_{j}^{t}$ broadly followed a gamma or Hoerl curve :

$$
\lambda_{j}^{t}=C(t)(j+1)^{A(t)}[B(t)]^{j+1},
$$

where $A(t), B(t)$ and $C(t)$ depend only on $t$. These preliminary plots appear (in conjunction with plots of the fitted model) in Section 5. From (3.8),

$$
\log \lambda_{j}^{t}=\log C(t)+A(t) \log (j+1)+(j+1) \log B(t) .
$$

It is evident from this last equation that the numerical parameters of the formula for incidence of risk may be estimated, for any particular $t$, by linear regression of the logged observations on incidence of risk against the variables $(j+1)$ and $\log (j+1)$. This has been done for each of a number of policy terms, and the results appear in Section 4. 
The estimation procedure described above produces estimates of the numerical parameters $A(t), B(t)$ and $C(t)$ separately for each policy term. Formulas have then been fitted to $A(t)$ and $B(t)$, treating these as functions of policy term $t$. Examples of the fitted Hoerl curves appear in the plots of Section 5.

Although regression has been used here to obtain estimates of the parameters $A(t), B(t)$ and $C(t)$, other forms of estimation are possible. It should be noted, however, in choosing an estimation criterion, that the empirical distributions of incidence of risk are incomplete for policies of terms exceeding 39 months, for the reason given in Section 2. Hence, any estimation method which requires the entire empirical distribution, such as method of moments, will not be feasible.

No modelling of $C(t)$ is necessary, since this parameter cancels in the quantity

$$
\lambda_{j}^{t} / \sum_{k=0}^{t} \lambda_{k}^{t}
$$

and so does not influence the earning of premium. Effectively, $C(t)$ is a parameter setting the level of claim frequency over the full term of the policy but not affecting its distribution over term.

Note that, if $j$ is regarded as continuous variable, then the gamma distributed variable, policy duration (months) at claim occurrence, will have a mean (see e.g. Hogg and Craig, 1970, Section 3.3),

$$
\begin{aligned}
& =M(t)[\text { say }] \\
& =[A(t)+1] /[-\log B(t)] .
\end{aligned}
$$

The parameters $A(t)$ and $M(t)$ are equivalent to $A(t)$ and $B(t)$ in characterizing the distribution of incidence of risk over policy term. In fact, $M(t)$ is a more convenient estimand than $B(t)$ since the former varies quite close to linearly with $t$. This is illustrated graphically in Section 4 .

Experimentation with the estimates of $A(t)$ as a function of $t$ suggests that it takes the following approximate form:

$$
A(t)=k+\exp (\alpha+\beta t),
$$

where $k, \alpha$ and $\beta$ are numerical constants.

Equation (3.11) can be reduced to the linear form

$$
\log [A(t)-k]=\alpha+\beta t,
$$

if the value of $k$ is known. In this case, the parameters $\alpha$ and $\beta$ can be estimated by linear regression. Simple simultaneous regression of $k, \alpha$ and $\beta$ is not possible however. Experimentation with trial values of $k$ indicated a value of -0.3 as suitable. It then follows from (3.10), (3.12) and the linearity of $M(t)$ that:

$$
\begin{gathered}
\log [A(t)+0.3]=\alpha+\beta t, \\
M(t)=\gamma t,
\end{gathered}
$$


The linear expression for $M(t)$ in (3.14) has been forced through the origin. On purely logical grounds, it is reasonable to assume that the average policy duration at claim occurrence must approach zero as the term of the policy approaches zero. As the relevant graph of Section 4 shows, this assumption is supported empirically. Estimates of the parameters $\alpha, \beta$ and $\gamma$ may now be found by regression of:

(a) the estimates of $\log [A(t)+0.3]$ on $t$; and

(b) the estimates of $M(t)$ on $t$, forced through the origin.

The numerical detail derived from performing the procedures described above is given in Section 4 .

\section{NUMERICAL DETAIL OF MODEL}

The first set of regressions described in Section 3.2, fitting separate distributions of incidence of risk to various terms $t$ yielded the following estimates of parameters of the Hoerl curve (3.8).

\begin{tabular}{cccc}
\hline & \multicolumn{3}{c}{ Parameter estimate } \\
\cline { 2 - 4 } Policy term & $A(t)$ & $B(t)$ & $\begin{array}{c}\text { Fitted mean policy } \\
\text { duration } M(t) \text { at } \\
\text { claim occurrence }\end{array}$ \\
\hline Months & & Months \\
12 & 1.127 & 0.700 & 5.96 \\
18 & 1.171 & 0.772 & 8.40 \\
24 & 0.501 & 0.857 & 9.86 \\
36 & 0.224 & 0.917 & 14.15 \\
48 & -0.054 & 0.959 & 22.51 \\
60 & -0.165 & 0.977 & 35.97 \\
72 & -0.247 & 0.977 & 33.06 \\
\hline
\end{tabular}

In this table, the values of $M(t)$ have been computed from $A(t)$ and $B(t)$ according to (3.10).

The following graphs exhibit the forms $A(t)$ and $M(t)$ as functions of $t$. They also display the curves fitted to these values by means of (3.13) and (3.14). The parameters $\alpha, \beta$ and $\gamma$ in those equations have been estimated as:

$$
\begin{aligned}
& \alpha=1.21014, \\
& \beta=-0.05531, \\
& \gamma=0.49063 .
\end{aligned}
$$



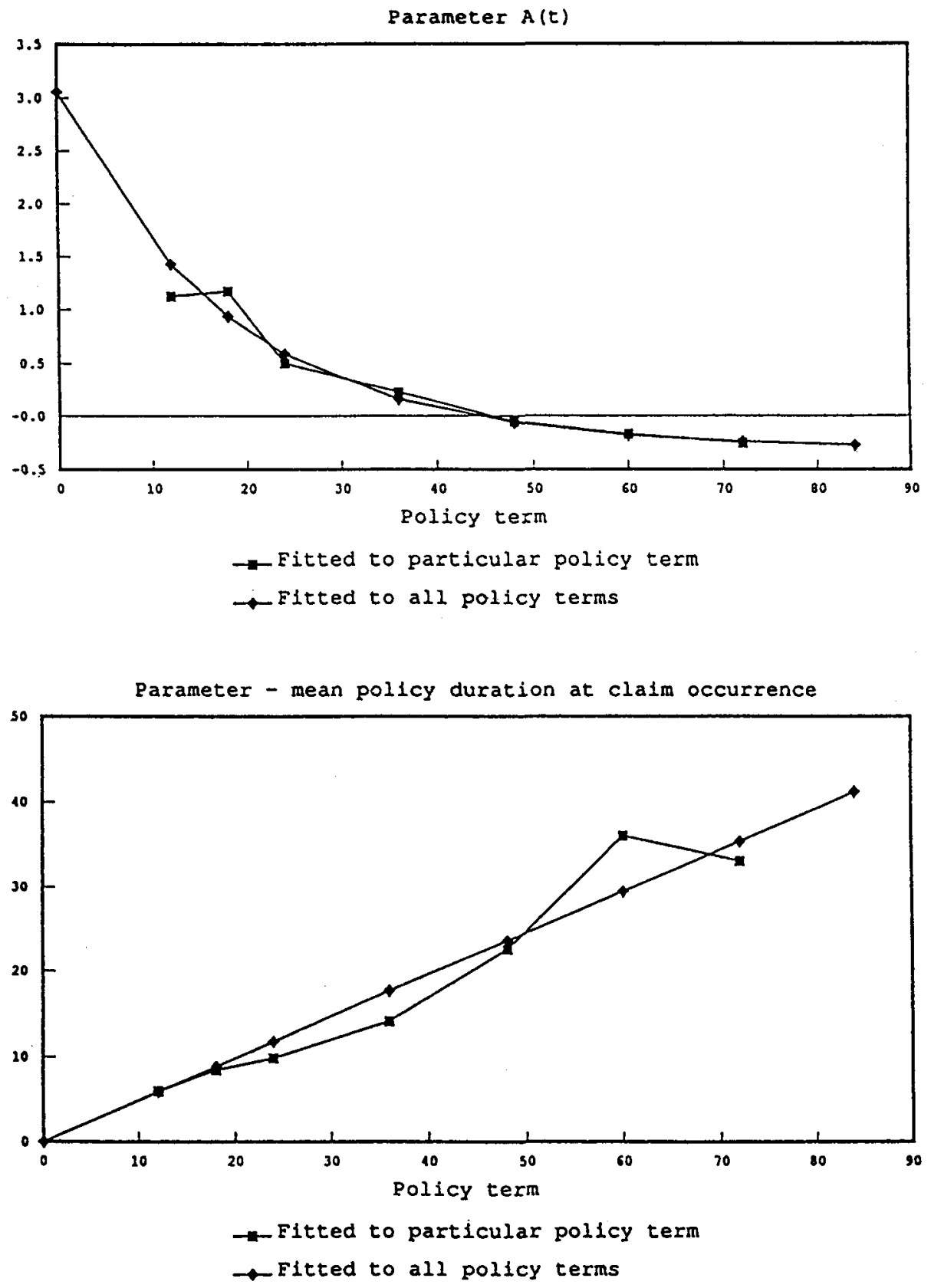
Thus, by insertion of these numerical values in (3.8), (3.13) and (3.14), the final model of incidence of risk is:

$$
\begin{aligned}
\lambda_{j}^{t} & =C(t) \times(j+1)^{\exp [1.21014-0.05531 t]-0.3} \\
& \times \exp \left[\frac{-(j+1)\{\exp [1.21014-0.05531 t]+0.7\}}{0.49063 t}\right] .
\end{aligned}
$$

It may be noted here that (4.1) involves a small bias. This arises from the informal procedure of logging the data, fitting a linear model and then exponentiating the fit. The effect of this can be seen as follows.

Suppose $X$ is a lognormal random variable:

$$
\log X \sim N\left(\mu, \sigma^{2}\right)
$$

whence

$$
E[X]=\exp \left(\mu+1 / 2 \sigma^{2}\right) .
$$

Let $\hat{\mu}$ be an estimator of $\mu$ such that

$$
\hat{\mu} \sim N\left(\mu, v^{2}\right)
$$

and estimate $E[X]$ by $\xi=\exp (\hat{\mu})$. Then

$$
E[\xi]=\exp \left(\mu+1 / 2 v^{2}\right)=E[X] \exp 1 / 2\left(v^{2}-\sigma^{2}\right) .
$$

This means that, as an estimator of $E[X], \xi$ is biased by a factor of $\exp 1 / 2\left(v^{2}-\sigma^{2}\right)$. Such bias arises in the inversion of both (3.9) and (3.13).

In the former case, constant factor biases such as this cancel out of the incidence of risk as defined in Section 3.1. In the latter case, however, a positive or negative bias in $A(t)$ will arise, with corresponding distortion of the resulting estimated incidence of risk. While correction of this distortion is possible by means of estimates of $\sigma^{2}$ and $v^{2}$, it is small in the example of this section and has been disregarded.

It is appropriate at this point to interpolate a couple of remarks in interpretation of the above model. The basic Hoerl curve (3.8) is characterized by a location parameter $M(t)$ and a shape parameter $A(t)$.

The empirically justified form (3.14) of $M(t)$ means that, on average, a constant proportion of policy term has elapsed at claim occurrence, whatever the term of the policy. If $A(t)$ did not vary with $t$, the last observation would mean that all incidence of risk distributions would be the same except for a change of scale by reference to $t$.

However, $A(t)$ does in fact vary with $t$. Effectively, $A(t)$ relates mode and mean since

$$
\begin{aligned}
\text { mode } & =\text { mean } \times[1+1 / A(t)]^{-1}, \quad \text { if } \quad A(t)>0 \\
& =0, \text { otherwise. }
\end{aligned}
$$


Thus, the smaller is $A(t)$, the smaller is the mode as a percentage of the mean. The above graph of $A(t)$ indicates that the mode (relative to the mean) shifts left with increasing policy term, reaching zero (policy attachment) when the policy term reaches about 4 years.

The exponential form of $A(t)$ as a function of $t$ is quite empirical, though any other form would need to observe the fact that (3.8) requires $A(t)$ to be bounded below by -1 .

\section{COMPARISON OF MODEL WITH EXPERIENCE}

The following three graphs provide illustrative comparisons of the experienced incidence of risk over policy term with that modelled. The three examples deal with a range of policy terms, from short (12 months) through the common medium term of 36 months to long (72 months).

Each graph, which corresponds to a particular $t$, displays three curves representing different functions over $j$. These functions are:

(a) the empirical incidence of risk defined by (3.7);

(b) the fitted incidence of risk (3.8), where fitting is by reference to just the empirical curve appearing in the graph, i.e. with fitting by reference to only the particular $t$ to which the graph applies (the parameters $A(t)$ and $B(t)$ are obtained from the table set out in Section 4);

(c) the fitted curve obtained from the final model (4.1), which applies to all values of $t$ and $j$.

The curves (a), (b) and (c) are designated in each graph "actual", "fitted" and "model" respectively. Each curve has been normalized so that the sum of its masses is unity since, as remarked just before (3.3), this indicates the distribution of incidence of risk required for premium earning purposes.

INCIDENCE OF RISK FOR POLICY TERM 12 MONTHS

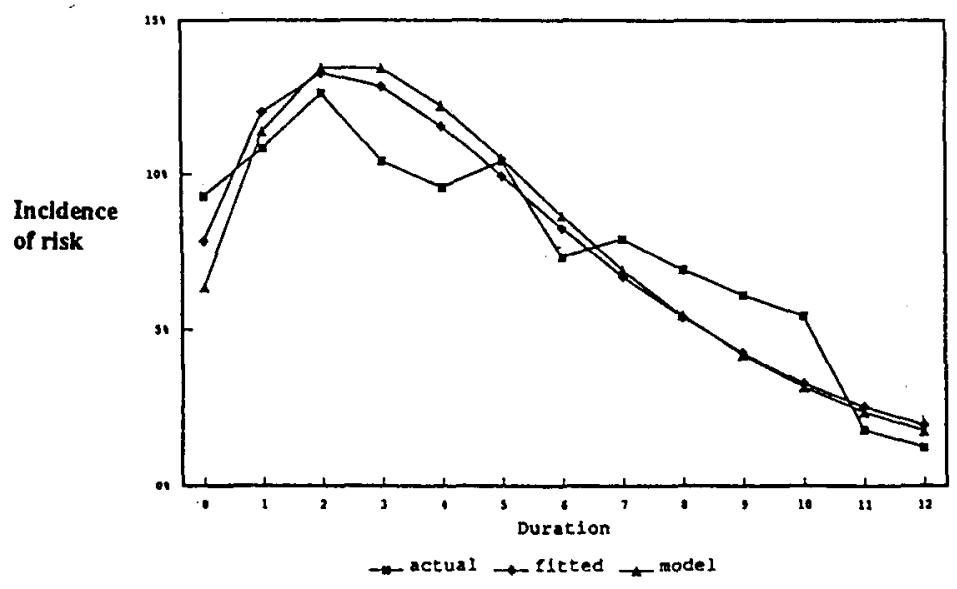




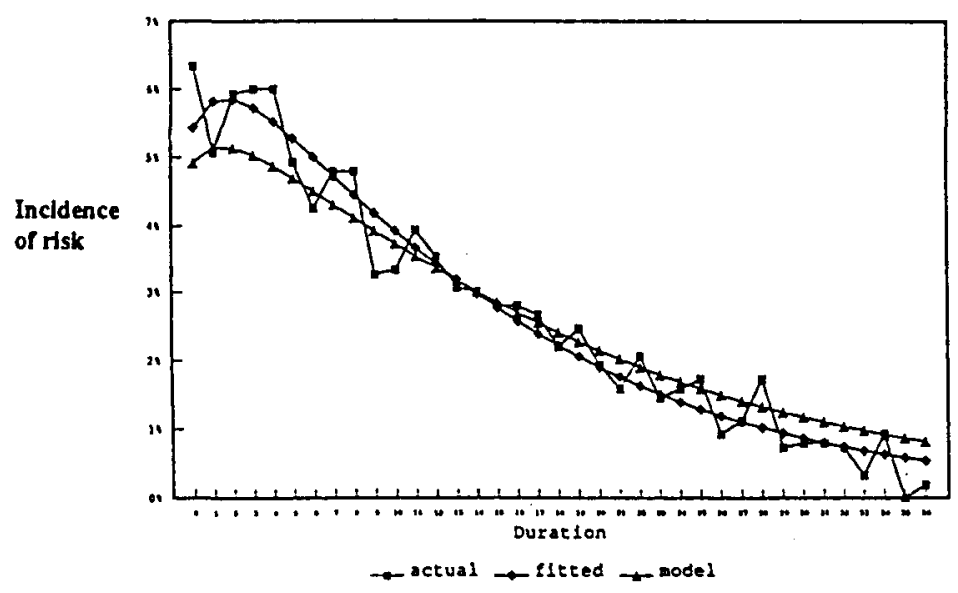

INCIDENCE OF RISK FOR POLICY TERM 72 MONTHS

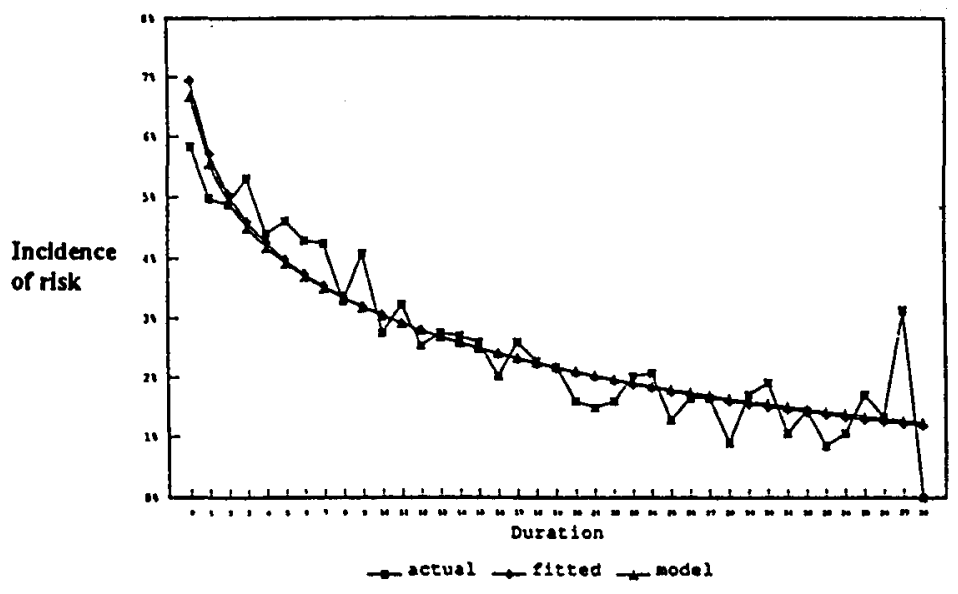

The three graphs are representative of the efficiency of the model generally. It is seen to fit the data quite well, though perhaps with a slight tendency to understate earned premium, the feature being particularly noticeable at term 36 months.

This rather minor inefficiency needs to be assessed in the light of the fact that the many values of $\lambda_{j}^{t}$ have been described with considerable parsimony in the final model (4.1), which depends on only four parameters. 


\section{EXTENSIONS AND QUALIFICATIONS}

\subsection{Estimation procedure}

The estimation procedure described in Section 3 is to some extent ad hoc. It has the advantage of simplicity since each of the two stages of the procedure involves simple univariate regression. Because of this, the fitting may be carried out within a spreadsheet.

Although Section 5 suggests that it produces reasonable results, it might be preferable from a theoretical point of view to fit an expression of the form (4.1) to the data by some recognized optimization procedure such as maximum likelihood, instead of by the two-stage procedure used in Section 3.2.

Estimation by this means would of course be considerably more difficult. It is not possible to linearize (4.1) in all its parameters simultaneously and so formal methods of parameter estimation would need to involve non-linear computation. This has not been attempted.

The objective stated in Section 1 was effectively the fitting of an incidence of risk model appropriate to all policy terms and dependent on a small number of parameters. This is to be contrasted with a situation of searching for a model whose parameter set is reduced just to the point where predictive power of the model is optimized.

The latter case would be dealt with by optimization of some performance measure such as the Akaike information criterion. This statistic has not been computed here.

\subsection{Economic conditions}

The model (4.1) has been obtained from data summarizing past experience. Use of this model for premium earning purposes amounts to forecasting the future incidence of risk under policies now in force. This in turn involves an implicit assumption that the future incidence of risk will be a reproduction of the past.

Credit insurance is likely to be sensitive to economic conditions, particularly when it provides unemployment coverage. There is, therefore, a need for caution in applying the earning model (4.1). The reserve for unearned premium implied by this model should be supplemented by a margin for adverse experience if economic circumstances suggest this to be warranted.

\subsection{Mortgage insurance}

Mortgage insurance represents to some extent an extreme case of credit insurance, extreme in the sense that the sums insured involved are generally much larger and the policy terms much longer than is the case under conventional credit insurance. 
The similarity between credit and mortgage insurance suggests that some of the ideas and functional forms used in this paper may be transportable to the latter line of business. As yet, no experimentation has been carried out in this area.

\section{REFERENCE}

Hogg, R. V. and CRAIG, A. T. (1970) Introduction to mathematical statistics. 3rd ed. The Macmillan Company, New York.

GREG TAYLOR

December 1991, Revised May 1993. 